Revue européenne des sciences sociales

European Journal of Social Sciences

XXXIX-121 | 2001

L'acteur. Un concept sur la scène des sciences

sociales

\title{
Villes, agents et acteurs en géographie
}

\section{Denise Pumain}

\section{(2) OpenEdition}

\section{Journals}

Édition électronique

URL : http://journals.openedition.org/ress/649

DOI : $10.4000 /$ ress.649

ISSN : 1663-4446

Éditeur

Librairie Droz

\section{Édition imprimée}

Date de publication : 1 novembre 2001

Pagination : 81-93

ISBN : 2-600-00663-X

ISSN : 0048-8046

Référence électronique

Denise Pumain, "Villes, agents et acteurs en géographie », Revue européenne des sciences sociales [En ligne], XXXIX-121 | 2001, mis en ligne le 11 décembre 2009, consulté le 20 avril 2019. URL : http:// journals.openedition.org/ress/649; DOI : 10.4000/ress.649 


\title{
Denise PUMAIN
}

\section{VILLES, AGENTS ET ACTEURS EN GÉOGRAPHIE}

\begin{abstract}
Véronica Noseda et Jean-Bernard Racine ont relaté l'itinéraire de recherche de géographes qui accordent un intérêt croissant à l'acteur, illustrant l'une des démarches qui justifient les conclusion de Jean-Pierre Gaudin quant à un probable «retour de l'acteur» dans les sciences sociales contemporaines. D'autres géographes, qui situent leurs travaux dans une problématique systémique, continuent cependant d'explorer d'autres voies, plus proches de la recherche de «structures objectives » et du dialogue entre sciences sociales et sciences de la nature, et qui suggèrent des «allers-retours » des acteurs aux systèmes. Plutôt que d'argumenter dans l'abstrait, on a choisi d'illustrer quelques questions soulevées par une telle démarche à propos d'un exemple particulier d'objet géographique, les systèmes de villes.

On interroge d'abord les premières tentatives de construction disciplinaire de ces objets complexes, afin de préciser quel rôle y jouent les acteurs et comment leurs interventions y sont représentées. On questionne ensuite des représentations plus récentes, qui formalisent les systèmes de villes en termes d'objets complexes évolutifs, en évaluant les performances et les contraintes des outils de modélisation dynamique et de simulation par les systèmes multi-agents associés à ces représentations. On propose une vue critique du paradigme des théories de l'autoorganisation, qui tout à la fois intègrent le niveau des acteurs et dispensent de leur observation précise, à certaines échelles d'analyse. On évoque enfin des pistes de recherche susceptibles d'articuler à chaque niveau les logiques propres des systèmes et des possibilités de leur inflexion par les acteurs.
\end{abstract}

\section{TROIS INTERPRÉTATIONS DES SYSTÈMES DE VILLES : L'ACTEUR DÉMIURGE, LA MAIN INVISIBLE OU L'ENTITÉ COLLECTIVE AGISSANTE}

Il faut rappeler d'abord la spécificité de la posture de la géographie parmi ce qu'il est convenu depuis quelques décennies d'appeler «les sciences de la ville». La géographie est à peu près la seule discipline des sciences sociales qui, avec quelques historiens du temps long et des spécialistes de l'aménagement du territoire, se donne pour projet d'expliquer non seulement «la » ville, mais aussi «les» villes, ces villes étant alors envisagées, pas uniquement dans leur diversité, spatiale et temporelle, fonctionnelle, morphologique ou culturelle, diversité qui inspire les explications comparatistes, mais encore d'après les ensembles qu'elles constituent du fait de leurs relations et de leurs interdépendances dans un territoire 
donné. Le plus souvent, les régularités observables dans ces ensembles de villes ne sont pas connues du public, et bien des acteurs «urbains» n'ont qu'une conscience très floue de leurs propriétés.

L'objet d'analyse construit par les géographes est formé de l'ensemble des villes qui occupent un même territoire et qui ont entre elles des possibilités de relations, plus ou moins fréquentes et intenses. Ces relations entre les villes prennent la forme d'interactions, sociales et spatiales, qui les rendent interdépendantes pour leur fonctionnement et dans leur évolution. De tels objets, repérables à l'échelle de territoires suffisamment vastes, ont été désignés par les observateurs dès le milieu du XIXe siècle sous les expressions de «réseaux urbains » ou «systèmes de villes ». Ces objets sont caractérisés par des propriétés qui les rendent en grande partie semblables ou comparables, d'un territoire à un autre (en général les territoires considérés sont des Etats du monde, ou des régions bien identifiées). Les propriétés communes aux systèmes de villes consistent en régularités statistiques, dans l'espacement des villes, dans les inégalités de leurs tailles, dans leur différenciation fonctionnelle et dans les cohérences de leur évolution.

Les interprétations successives proposées pour expliquer ces régularités les font assez peu dépendre de l'intervention explicite d'acteurs identifiables. Dans les théories classiques, le réseau urbain est plutôt donné comme l'expression directe de logiques d'intentionnalité, qui auraient joué à cette échelle macro-géographique du territoire pris dans son ensemble, comme si un acteur avait maîtrisé dans sa totalité la construction de l'armature urbaine du territoire. Ainsi, le passage d'une échelle à l'autre n'est le plus souvent pas explicité, comme s'il n'y avait pas de hiatus entre l'organisation produite à l'échelle territoriale et les intentions d'acteurs individuels. La logique de composition est donc en général absente des modèles issus de ces théories. Trois grands schémas interprétatifs ont été développés parallèlement, depuis la fin du XVIIIe siècle, et jusque vers les années 1970 (Pumain, Robic, 1996).

\section{Des intentions de contrôle, globales ou locales}

Le premier explique les propriétés des ensembles de villes par la volonté ou la nécessité de «couvrir le territoire», pour les besoins de sa défense, de son encadrement administratif, ou de la desserte des populations qui l'habitent en biens et en services. Des réseaux de places fortes à la Vauban à la théorie des lieux centraux de Christaller, l'approche est toutefois différente en termes d'échelle de représentation de l'intentionnalité: alors que la première lie explicitement la disposition des places à la volonté royale d'organiser la protection du «pré carré », la seconde considère qu'une configuration spatiale optimale des lieux centraux se réalise en raison d'un double choix (ou plutôt d'une double nécessité, ou contrainte) des acteurs en présence à l'échelon le plus local. En se rendant au lieu central (de consommation) le plus proche, les consommateurs minimisent leur dépense de transport pour se procurer un service ou un bien de niveau donné, tandis que les producteurs de biens ou de services s'installent partout où il leur est possible de trouver une clientèle suffisante, dans le rayon d'action (la portée) du bien ou du service qu'ils offrent. Les «acteurs» en question sont en fait plutôt des «agents " économiques, au comportement moyen stéréotypé. Le regroupement des biens et des services d'un niveau donné dans les mêmes centres urbains est un 
postulat de la théorie, qui sera relié plus tardivement à un comportement d'acteur (par le biais des économies d'agglomération). La spatialisation de l'équilibre offre/demande, par une hiérarchie emboîtée de lieux centraux offrant des biens de niveau et de portée différents localisés aux sommets d'hexagones réguliers, est ingénieuse, mais la manière dont s'opère le passage entre les comportements individuels des agents et la réalisation d'une configuration satisfaisant à des critères d'optimisation à l'échelle macroscopique est suggérée plus que démontrée. On projette en fait sur le niveau macroscopique, pour l'expliquer, des intentions d'échelle individuelle, alors même qu'aucun opérateur n'est capable d'intervenir à l'échelle macroscopique du réseau urbain. De la rationalité du comportement des acteurs locaux découlerait la rationalité du système des villes pour la couverture du territoire.

\section{L'émergence régulière des étapes dans les réseaux de circulation}

Une seconde approche, tout aussi récurrente que la première, n'a pas été aussi loin dans les tentatives de formalisation, mais était porteuse d'autres ouvertures épistémologiques. Cette famille d'explications s'appuie sur un type de fonctions des villes, hôtelières et marchandes, qui en font des étapes dans des réseaux de circulation. Cette explication pourrait avoir été intégrée dans la précédente, mais son intérêt est d'avoir été très tôt formulée en des termes favorisant une interprétation évolutive des réseaux urbains. C'est Elisée Reclus notamment qui relie la taille des villes à leur espacement, le long des «courants principaux de la vie nationale »: « on a constaté que les villes les plus importantes de ces grandes voies sont situées en général à deux étapes les unes des autres, tandis que les localités moins considérables marquent les étapes intermédiaires, et des bourgades moins importantes encore les lieux d'arrêt momentané. Cette distribution régulière des agglomérations humaines sur les grandes routes de guerre et de commerce s'explique par les anciens modes de locomotion; piétons et cavaliers avaient à scander leur marche, et les villes s'accroissaient en raison du temps de séjour» (Reclus, 1877). Les plus grandes villes, plus espacées, seraient donc celles qui auraient gardé leur rôle transactionnel tout au long des progrès des techniques de transport. L'intention explicite des acteurs que sont les constructeurs puis les opérateurs de réseau est rarement évoquée, dans des interprétations qui se limitent le plus souvent à l'effet mécanique de l'accroissement de la vitesse des transports sur l'allongement des étapes.

\section{Etres géographiques ou acteurs collectifs}

La troisième famille d'interprétations tend à faire de la ville, sinon un acteur collectif, du moins un organisme autonome qui prend place dans un ensemble de villes où se réalisent des complémentarités entre des spécialisations urbaines variées. Ces représentations ouvrent la voie à des formulations systémiques. La plus aboutie est sans doute celle proposée par H. Mackinder (1902), qui distingue en Angleterre trois générations de villes, depuis les nombreux petits marchés locaux, à peu près auto-suffisants mais subordonnés par rapport au grand foyer centralisateur de Londres, puis les villes industrielles, nécessairement spécialisées et complémentaires les unes des autres, enfin d'autres spécialisations (tourisme, 
résidence, transport) non plus liées à des sites de ressource mais à des types de situations dans l'ensemble urbain. Les expressions de «conurbations» de P. Geddes ou de «fédérations urbaines» proposée par Mackinder correspondent à l'identification de systèmes coordonnés de peuplement, différenciés fonctionnellement, dans une conception évolutive inspirée de l'évolution des espèces et guidée par le progrès technique. L'identification implicite de la ville-organisme à un acteur vient facilement sous la plume des géographes, quand Vidal de la Blache expose quel est le «rôle» de la «ville régionale», ou encore quand Pierre George résume l'inversion des relations qui confère à la ville une fonction organisatrice et motrice dans l'espace géographique:« désormais, ce n'est plus la région qui fait la ville, mais la ville qui fait la région ». Cependant, l'intuition ne va guère au-delà de la métaphore, l'image de la ville en tant qu'acteur collectif n'a jamais été vraiment formalisée.

Compte tenu du caractère immédiatement peu perceptible du niveau du système des villes, aux échelles d'observation du fait urbain habituelles dans la plupart des sciences sociales, il est assez peu surprenant que l'articulation entre le niveau des acteurs, celui de la ville et celui des systèmes de villes ne se soit formulée que lentement. Le paradoxe de cette histoire trop brièvement retracée de la représentation des systèmes de villes est que les tentatives qui étaient les plus abouties pour expliciter le lien entre des motivations ou des comportements d'acteurs ou d'agents et la configuration macro-géographique ont joué un rôle bloquant, en limitant l'explication à la compréhension d'un équilibre statique, à la description de l'état du système des villes à un moment donné, tandis que les explications de nature plus holiste, qui admettaient l'autonomie relative de l'objet ville dans sa persistance historique, ont ouvert la voie à des représentations formelles dépourvues d'acteurs, mais qui permettent de mieux expliquer à la fois la structure du système des villes et son évolution.

\section{POUR SIMULER LA DYNAMIQUE DES VILLES EN SYSTÈME: DES AGENTS SANS ACTEURS}

C'est en 1964 que Brian Berry propose une expression très synthétique «cities as systems within systems of cities», mais la transcription de ce que cette formule implique en termes de formalisation théorique et de modèles n'interviendra que plus tard, à partir de travaux d'observation empirique de l'évolution des systèmes de villes (notamment ceux de A. Pred aux Etats-Unis et en France de l'équipe P.A.R.I.S.) ainsi que par des échanges avec des spécialistes de sciences physiques et d'informatique.

\section{Un système à trois niveaux d'observation}

On propose une représentation du système des villes à trois niveaux d'observation: le niveau individuel, celui des acteurs urbains, les décrit par des variables d'état significatives, par exemple leur position dans le cycle de vie, leur profession ou leur revenu, leur pouvoir d'intervention. Le niveau de la ville peut être simplement décrit par l'addition, l'agrégation de ces acteurs individuels (par exemple à travers un descripteur qui dénombre la population totale de la ville) 
mais le plus souvent les variables d'état qui sont caractéristiques de ce niveau ne peuvent pas être définies à l'échelle des acteurs (par exemple une densité de population). Elles sont cependant produites par des interactions entre les acteurs, qui réalisent par exemple un gradient de prix fonciers, un type de morphologie sociale urbaine, ou une organisation politique (un jeu d'acteurs comme le célèbre SIMCity par exemple met bien en évidence cette dépendance entre l'intervention des acteurs et la forme prise par l'organisation urbaine). Même une variable d'état obtenue par agrégation comme la taille de la ville exprime aussi des processus d'accumulation sur le temps long et de filiation entre des activités qui déterminent plus qu'une simple totalisation, un niveau de complexité plus ou moins important de la société et des fonctions urbaines. L'important est que l'émergence de ces structures, qui explique que l'on fasse appel à de nouveaux descripteurs, définit un objet «ville», un système persistant dans le temps et qui peut être observé de façon relativement autonome par rapport aux acteurs individuels qui l'ont construit ou qui y vivent et en vivent, en tenant compte nécessairement des «contraintes» que représente pour eux l'existence de cette organisation. Cette représentation de la ville comme système d'acteurs en interaction paraît toutefois plus pertinente pour décrire des processus d'intérêt local et de court terme (par exemple organisation des modes de transport, choix urbanistiques, conflits pour l'usage du sol...) que pour comprendre le devenir d'une ville, à moyen et à long terme, lequel dépend beaucoup plus d'interventions externes à la ville (par exemple du fait des choix de localisation de grandes entreprises).

Si la ville peut être assez bien délimitée mais a assez peu d'autonomie en tant que système contrôlant sa propre évolution, l'inverse peut être suggéré pour le système des villes: son comportement révèle d'importantes cohérences, mais il est très difficile de lui assigner des limites et de définir quel est son environnement. Souvent, on définit un système de villes par son enveloppe territoriale, régionale ou nationale, dans la mesure où les conditions sociales définies dans ce cadre assurent une certaine homogénéité de fonctionnement et solidarité de devenir. La difficulté de délimiter un système de villes, de séparer un espace où les interactions internes sont plus fortes que les échanges externes, est en fait liée aux propriétés de la structure hiérarchique de ces systèmes: en moyenne, la portée des interactions varie de manière systématique avec la taille des villes, la part des échanges à grande distance est plus grande dans les villes les plus importantes. Il faut donc concevoir un système de villes non seulement comme un système ouvert, mais avec une ouverture d'autant plus large que les villes éléments du système sont grandes. On peut reprendre provisoirement la définition d'A. Pred: « un système de villes est défini comme un ensemble national ou régional de villes qui sont interdépendantes, de telle façon que toute modification significative dans les activités économiques, la structure sociale, le revenu ou la population de l'une d'entre elles contribuera directement ou indirectement à modifier les activités économiques, la structure sociale, le revenu ou la population d'une ou de plusieurs autres villes membres du système» (Pred, 1977, p.13). Remarquons que les propriétés globales de l'objet ville qui sont utilisées pour expliquer la formation du troisième niveau pertinent d'observation, celui des systèmes de villes, constitué de villes en interaction forte les unes avec les autres, ne sont généralement pas les mêmes que celles qui caractérisent la ville comme produit d'interactions de ses acteurs: la taille de la ville, sa fonction économique, sa situation géographique, 
sont généralement parmi les indicateurs retenus pour analyser les interactions entre les villes, qui expliquent l'émergence de propriétés nouvelles, au niveau du système des villes, comme une structure fortement hiérarchisée, une trame spatiale régulière, des complémentarités fonctionnelles.

\section{Un modèle stochastique de la croissance des villes}

Une représentation en trois niveaux d'organisation apparente le système des villes à d'autres «systèmes hiérarchiques ouverts» observables dans la nature. Constitués de corpuscules élémentaires en très grand nombre, qui se réunissent en sous-systèmes par des processus de nature variable, ces systèmes ont en commun une différenciation hiérarchique de la taille des sous-systèmes suivant une loi de type parétien. On démontre que cette structure peut s'expliquer par des modalités de répartition de la croissance des sous-systèmes qu'il est possible de résumer par des modèles très simples, tel celui de Gibrat (1931) pour les villes. Selon ce modèle stochastique, les villes d'un même système croissent en moyenne selon un taux de variation commun, assorti de fluctuations réparties aléatoirement dans l'espace et dans le temps. Cela suffit en théorie à expliquer les très grandes inégalités de la taille des villes, et la persistance du rang des villes dans la hiérarchie, à moyen et même sur le long terme. De telles modalités d'évolution correspondent aussi aux observations, et peuvent être rapprochées de représentations plus qualitatives qui mettent en jeu la concurrence entre les villes pour la captation des innovations.

\section{Modélisation de l'évolution par les systèmes multi-agents}

L'expérimentation des modèles stochastiques de croissance, qui confirme la validité assez générale de cette forme d'explication, révèle cependant des écarts systématiques avec les observations, qui incitent à introduire des hypothèses un peu plus complexes, notamment pour rendre compte du caractère très généralement hypertrophié de la taille des villes qui sont à la tête des réseaux urbains, et aussi de l'accroissement tendanciel des inégalités entre les villes au cours de l'histoire, notamment pendant les phases d'urbanisation intense. Le modèle est aussi impuissant à rendre compte des croissances fortes de certaines villes du fait de l'apparition de nouvelles spécialisations économiques, et donc des bifurcations qu'elles entraînent dans la structure du système des villes. Il faut donc compléter la description des villes, en ajoutant à leur taille des indications sur la nature de leurs activités notamment. Des modèles dynamiques non linéaires exprimés par des équations différentielles ont été expérimentés, mais ils ne laissent guère de souplesse dans la simulation des interactions spatiales, des relations entre les villes. On a donc entrepris de construire un modèle à partir d'outils informatiques, issus des techniques d'intelligence artificielle et fondés sur des méthodes de programmation «orientées objets ». Ces «systèmes multi-agents» sont pour nous plus que de simples instruments de simulation, substitut de l'expérimentation en sciences humaines, car certaines des représentations dont ils sont porteurs sont compatibles avec celles mises en œuvre dans la définition des systèmes de villes. Nous ne donnerons ici qu'une intuition de ce qui relève, à chaque fois qu'une nouvelle forme de modélisation est expérimentée, d'une opération dite de «transfert» de concepts et de modèles. 
Un agent est «une entité réelle ou abstraite qui est capable d'agir sur ellemême et son environnement»(Ferber, 1995). C'est un outil informatique de modélisation doté de caractéristiques et de règles de comportement. Un agent fait partie d'un système dans le sens où il échange des informations avec d'autres agents, qui sont susceptibles de modifier son propre état ou d'activer certaines de ses règles de comportement. Les agents sont regroupés en classes, dont les membres partagent les mêmes propriétés. Une particularité de la modélisation par les systèmes multi-agents, appelée «héritage», conduit à organiser les classes d'agents selon un arbre hiérarchique, les agents d'une classe de niveau supérieur «héritant» de toutes les propriétés de celles du niveau inférieur, en y ajoutant quelques aptitudes ou règles supplémentaires. Une telle organisation de la connaissance sur les agents nous a intéressés, car elle permet une transposition, commode parce que très directe, de la structure hiérarchique des systèmes de villes, de leur différenciation par niveaux de complexité, sociale et fonctionnelle.

Tout à fait comme dans les théories de l'auto-organisation qui ont inspiré la construction de modèles dynamiques, le fonctionnement des systèmes multiagents suppose que la structuration du système à l'échelle macroscopique résulte seulement des interactions entre les agents du niveau microscopique. Il n'y a pas dans le modèle de paramètre de contrôle global, d'optimum à réaliser, pas d'intervention qui jouerait directement à l'échelle macrogéographique. Les interactions sont «locales », entre agents voisins, mais on peut en faire varier la portée spatiale, afin de reproduire les inégalités des rayons d'intervention des villes (leur accessibilité dans les réseaux dépend de leur taille) et leur élargissement au cours du temps. Le modèle définit des processus de production et d'accumulation de la population et de la richesse, de leur répartition entre les villes, et de l'acquisition par celles-ci de niveaux de fonction plus ou moins complexes, susceptibles de renforcer leur pouvoir attractif et leur aptitude à créer de l'innovation. Pour des durées longues de l'histoire des villes, sur quelque 2000 ans d'histoire, le modèle permet de reproduire les propriétés macroscopiques de la structure et de l'évolution des systèmes de villes, la croissance distribuée dans tout le système étant liée à la concurrence entre les villes pour la captation des richesses produites dans le territoire et par elles-mêmes, tandis que des effets cumulatifs de sélection hiérarchique et de spécialisation fonctionnelle se produisent sous l'effet de la diffusion hiérarchique des innovations et de la contraction de l'espace-temps (Bura et al., 1996).

Différents tests sont en cours qui doivent permettre de vérifier l'aptitude du modèle à produire des systèmes de villes de configuration différente (par exemple très macrocéphale comme en France ou moins centralisé comme en Europe rhénane) ou ayant eu des séquences de développement décalées et d'intensité inégale (par exemple entre pays développés et Tiers monde). Une autre hypothèse intéressante pour des simulations concerne l'émergence de réseaux supranationaux de villes spécialisées, dont l'évolution dépendrait davantage des connections qu'elles ont entre elles (hypothèse de l'économie d'archipel de P. Veltz) que de leurs relations dans leur territoire d'appartenance. On fait l'hypothèse que de simples changements de valeurs de paramètres peuvent résumer ces différences qualitatives dans l'émergence des systèmes de villes. On suppose aussi que non seulement le modèle peut reproduire l'évolution observée sur le temps long d'un système, mais encore permet des projections de son état probable à quelques 
décennies d'intervalle, sans qu'il soit nécessaire, à cette échelle de temps et d'espace, de faire intervenir explicitement des acteurs individuels.

\section{FAUT-IL RÉINTRODUIRE LES ACTEURS?}

La simulation des systèmes de villes par des modèles statistiques simples ou au moyen de systèmes multi-agents ne prétend pas pouvoir expliquer toutes les particularités de la structure et de l'évolution de ces systèmes. Elle donne des cadres pour les comprendre, les simulations produisent des trajectoires de système, plausibles ou fictives. En faisant comme s'il n'y avait pas d'acteur, individuel ou institutionnel, qui soit capable d'infléchir cette évolution, elles permettent de relativiser, mais aussi de mesurer le pouvoir éventuel d'intervention des acteurs. L'assimilation d'une ville à un agent dans un système ne va cependant pas de soi. D'aucuns dénoncent la fonction de masque, voire la volonté d'occultation des acteurs «réels » que recouvrirait la figure de style, ou l'agrégation statistique, l'acteur collectif dépersonnalisé, qui tend à réifier un objet spatial en «être géographique».

\section{Rejet d'une géographie inhumaine, ou revendication du pouvoir des acteurs?}

Qu'on me pardonne la mention d'un souvenir personnel, aux fins d'illustrer quelle a pu être la réception par les géographes du pouvoir explicatif des modèles statistiques sans acteurs. Dans le contexte de très forte urbanisation des années 1960-70, de nombreuses recherches avaient été lancées pour identifier les facteurs de la croissance des villes. Une équipe coordonnée par Ph. Pinchemel et E. Dalmasso s'était penchée sur la liaison entre la construction des chemins de fer et l'urbanisation depuis le siècle précédent, avec le souci d'observer quel pouvait être le rôle des infrastructures dans le processus. Lorsque furent présentés les résultats de traitements statistiques, montrant qu'un modèle stochastique très simple rendait compte de la quasi totalité des inégalités de la croissance entre les villes et de l'évolution du système, et ce, indépendamment de toute intervention d'acteurs identifiés, les conséquences furent considérées avec une certaine perplexité (Pumain, 1982). Je me souviens encore de la remarque que fit l'un des géographes, pourtant des plus ouvertsparmi ceux de sa génération à l'idée de la modélisation: «c'est triste».

Pour bien d'autres, de telles recherches furent jugées inutiles, voire «dangereuses» (pourquoi ce terme, si souvent accolé aux modèles quantifiés, n'est jamais appliqué aux recherches de facteurs explicatifs purement qualitatifs?). Oser chercher, et trouver, des déterminations passant par des facteurs «objectifs », globaux et mesurables, comme la taille des villes, leur composition sociale ou leur situation géographique, sans passer par la révérence à l'action décisive de tel maire ou de tel entrepreneur, c'était «réifier l'espace» et, pour beaucoup, commettre l'impardonnable oubli des «hommes» dans l'analyse géographique.

Tout aussi déconcertantes sont les réactions de certains hommes politiques, à qui de tels résultats pourraient cependant apporter de précieuses informations quant aux marges de manœuvre dans lesquelles inscrire leur action. Même de fort 
éminents représentants de la DATAR ont préféré, à des estimations « réalistes » de la population des villes européennes projetées à des horizons de vingt ou trente ans, des scénarios beaucoup plus romantiques illustrant des situations contrastées bien plus excessives, toutes plus improbables les unes que les autres, mais jugées plus «parlantes» pour guider des choix politiques.

Dans les trois cas, on trouve le même respect, voire la fascination, pour l'acteur, le décideur, le démiurge, celui qui a le pouvoir d'écrire sur la surface de la terre, de laisser une trace identifiée pour les générations futures. Les hommes d'action, comme leurs observateurs, auraient-ils besoin de croire à leur efficience absolue, au caractère déterminant de leur décision? Cependant, qu'elles soient formulées au nom de la liberté de l'individu, de la richesse humaine de l'enquête ou de la capacité sociale du décideur, ces objections rejoignent la critique de l'individualisme méthodologique, selon laquelle un bon modèle doit se référer aux motivations des individus concernés. Il revient alors aux auteurs de modèles «agrégés » d'expliquer la portée de leurs représentations formelles, et surtout d'en approfondir la signification en enrichissant leur contenu, d'une manière qui produise plus de sens pour les acteurs.

\section{La présence implicite des acteurs dans les modèles d'auto-organisation}

Il faut d'abord lever un possible malentendu. Indiquer que «tout se passe comme si » les actions des acteurs n'étaient pas décisives ne signifie pas qu'il ne sert à rien d'agir. Dans un système où les villes sont en situation de concurrence les unes avec les autres, ce sont les multiples actions réalisées par les acteurs qui expliquent que toute ville appartenant au système a une probabilité très forte d'évoluer, quantitativement et qualitativement, comme les autres villes. Bien évidemment, les acteurs, la société, sont dans le système des villes, qui n'évoluerait pas sans eux! Mais on n'est pas obligé de les représenter explicitement dans un modèle conçu pour simuler l'évolution du système, parce que «tout se passe comme si » les multiples actions des acteurs individuels d'une ville pouvaient se résumer par un «comportement» global, comme si, avec une certaine probabilité, une loi statistique gouvernait le passage de la ville d'un état à un autre.

Remplacer l'action des acteurs par un processus d'évolution moyenne assortie de fluctuations aléatoires est une pratique courante dans les modèles de simulation. Dans ces modèles, le hasard intervient, non pas à titre d'hypothèse quant à l'irrationalité du monde ou à la neutralité du géographe qui l'interprète, mais comme une facilité de calcul, ou plutôt comme un moyen de réduire le coût de l'observation: chacun des comportements individuels ne pouvant être décrits dans leur spécificité et leur finalité, on simule leur variabilité par des fluctuations autour d'un comportement, sinon moyen, du moins typique. Au total, si l'on parvient à reproduire l'évolution de la structure macro-géographique avec une précision suffisante, on peut accepter à ce niveau de simuler par des processus aléatoires l'absence d'information où l'on se trouve quant à la diversité des acteurs.

Ainsi, considérer que les systèmes de villes évoluent selon une dynamique d'auto-organisation signifie bien que leur configuration et leur évolution résultent d'abord des interactions entre les acteurs qui y ont investi et qui appuient leur action ultérieure sur les ressources mobilisables localement. L'imitation de ce qui a été accompli ailleurs, l'innovation destinée à anticiper sur les concurrents, sont 
les principaux processus intervenant au niveau «microscopique» de la structure du système, celui des acteurs individuels, et qui se traduisent au niveau «macroscopique» dans des configurations structurelles et des dynamiques semblables, d'un système de villes à un autre, quels que soient les modes de fonctionnement politiques ou économiques des sociétés concernées. Les modèles d'auto-organisation permettent ensuite d'observer comment, dans certains cas, ces comportements peuvent produire, par leurs interactions, à des échelons géographiques supérieurs, des formes nouvelles, des structures non intentionnelles, dont le contenu n'est ni programmé ni même pensé par les individus. Cet «ordre par fluctuation» est-il si irréaliste? Le plus grand nombre des décisions qui sont prises et qui ont pour effet d'agir sur la structure du système des villes: décisions individuelles de migrer, décision d'implanter une entreprise, de bâtir des constructions..., le sont en dehors de toute planification autoritaire ou de toute volonté concertée, et le plus souvent en toute inconscience, pour la plupart de ses acteurs, de l'existence d'un «système» matérialisant les interdépendances entre les villes.

En revanche, ces actions des individus sont effectuées sous une contrainte forte: les acteurs individuels et collectifs qui composent le milieu social d'une ville ne sont pas indépendants mais connectés, informés mutuellement de leurs actions, et sont en concurrence avec ceux des autres villes pour capter les profits associés à l'exploitation des innovations. Les routes et relations qui unissent les villes d'un même système sont aussi des réseaux sociaux par lesquels transitent les informations. Plusieurs travaux historico-géographiques ont souligné le rôle considérable des échanges d'informations dans le développement des réseaux urbains. Une ville ne saurait évoluer indépendamment des autres villes, sans que ses acquis ne soient aussitôt imités ou contestés. La concurrence entre les acteurs localisés dans les villes, qui est le corollaire de la connexité des réseaux urbains, conduit par agrégation à une concurrence entre les villes du système des villes, et explique la métastabilité (trajectoire stable, ou équilibre dynamique) de la structure du système évoluant sous cette contrainte. C'est en particulier la concurrence entre les acteurs attachés aux différents lieux qui produit les importantes régularités structurelles, l'amortissement de la formation des différences par la diffusion de l'innovation, tandis que la très forte connexité du système contribue à en stabiliser l'évolution.

C'est ainsi que le système macro-géographique des villes, par la structure de différenciation des villes qui le caractérise, a des effets en retour sur les propriétés de chaque ville, lesquelles délimitent en partie les actions possibles pour ses acteurs. La position relative d'une ville dans le système est maintenue par la multiplicité des interactions entre les acteurs des différentes villes. Localement, au niveau méso-géographique de chaque ville, tout se passe comme si, alors que les réseaux sociaux se renouvellent par migration des personnes et d'une génération à l'autre, certains savoir-faire urbains se perpétuaient dans les mêmes lieux, ce qui ne peut se comprendre que par un ensemble d'effets en retour et de contraintes exercées par les lieux sur les acteurs, et par des processus d'apprentissage exigeant un temps assez long pour apporter des résultats significatifs dans la compétition urbaine. Il en résulte que, sans qu'on puisse l'assimiler conceptuellement à un acteur collectif, la ville s'identifie par des caractéristiques suffisamment stables et des régularités de transitions entre ses différents états, qui justifient qu'on la représente par un agent dans un système. 
Dans le système très fortement connecté que forment les villes, la taille des villes n'est pas une grandeur que l'on pourrait contrôler de l'intérieur d'une ville. Elle est le produit non intentionnel du réseau complexe des interactions qui se développent entre les villes, par les acteurs qui y investissent, économiquement, socialement et symboliquement. La course au gigantisme urbain n'a d'ailleurs pas à être toujours une règle du jeu assumée, elle peut être le résultat, plus ou moins aléatoire, plus ou moins intériorisé dans la conscience d'individus ou le programme de certains groupes, de stratégies d'imitation ou d'anticipation sur ce qui se fait ailleurs, dans d'autres villes. Nous pouvons alors reformuler la définition proposée par A. Pred: dans une perspective géographique, un système de villes est un ensemble, évolutif et ouvert, de villes interdépendantes dans un territoire, qu'il est pertinent d'observer à trois niveaux (les acteurs urbains, la ville, le système des villes). Caractérisée par une structure hiérarchique et une diversité fonctionnelle produites par une histoire, sa trajectoire dynamique est constituée par les interactions entre les villes, qui déterminent des ajustements permanents de leur état à des modifications suscitées par leur émulation, mais qui ne peuvent modifier la structure du système qu'en période d'instabilité (par exemple, au début ou à la fin d'un cycle d'innovations, ou à la suite de modifications de limites territoriales - la bifurcation peut alors être analysée comme provoquée par une perturbation «extérieure » au système).

\section{De l'intervention directe des acteurs}

L'intervention des décisions politiques dans l'établissement ou le développement des villes est souvent considérée comme très importante, et probablement surestimée. Certains insistent sur le rôle décisif des acteurs, fondateurs (ou parfois destructeurs) de villes - notons en regard l'échec de maintes fondations urbaines, surtout parmi les tentatives les plus récentes dans l'histoire. Certes, dans l'Antiquité, au Moyen-Age en Europe, ou encore au XIX ${ }^{\mathrm{e}}$ siècle dans certains pays de colonisation de peuplement, la sélection d'implantations urbaines a pu faire l'objet de décisions politiques ou administratives. Quelle qu'ait été et soit encore l'efficacité des interventions destinées explicitement à contrôler un territoire au moyen de l'implantation régulière de fonctions centrales, le plus souvent hiérarchisées, il peut être tenu pour certain qu'elles ne constituent pas cependant le déterminant essentiel de la structure et de l'évolution des systèmes de villes actuels. Au cours des temps historiques, les fluctuations (créations ou destructions de villes) liées à ces décisions se raréfient (Archaeomedes, 1998). Par ailleurs, des organisations volontaires des systèmes de villes se traduiraient nécessairement par une plus grande variété de formes, selon les régimes politiques et les systèmes économiques, et par des à-coups plus prononcés dans leurs évolutions.

Il reste que la variété des configurations des systèmes de villes dépend parfois assez directement des formes d'organisation du pouvoir territorial. Ainsi, en Europe, la centralisation politico-administrative du territoire français, qui a persisté sous différentes formes pendant plusieurs siècles, se traduit par une macrocéphalie du système des villes (le rapport entre le poids de la première ville et celui de la seconde est de 7 à 1 , contre moins de 4 à 1 en moyenne dans les pays développés) qui contraste avec la hiérarchie plus régulière des villes des régions rhénanes par exemple. Les interventions politiques ont tout au plus apporté 
quelques retouches à des structures qu'il est très difficile de faire évoluer. Ainsi at-on salué en France la politique des métropoles d'équilibre menée par la DATAR, qui certes n'a pas supprimé la primauté parisienne, mais qui a contribué à stopper la poursuite de cette concentration. Dans les pays d'Europe de l'Est, il a fallu cinquante années de politique socialiste d'équipement des villes moyennes pour renforcer un peu ce niveau de la hiérarchie, sans toutefois réduire la prééminence des capitales. L'implantation d'une nouvelle capitale au Brésil a permis de compléter l'armature urbaine du territoire, mais sans remettre en cause la prépondérance des grandes villes de la région côtière...

Une telle observation d'effet à la marge ne tend pas à disqualifier la politique et les actions d'aménagement du territoire, toujours nécessaires puisque entre des lieux en concurrence, les inégalités tendraient à s'accentuer sans intervention régulatrice. Mais il faut savoir que ces actions ont des limites (ou des coûts): on a pu ainsi en France accompagner le processus de diffusion hiérarchique des innovations corrélatif de l'urbanisation des Trente Glorieuses par les politiques successives des métropoles d'équilibre, des villes moyennes, puis des pays, on n'a pas pour autant reconfiguré le système des villes françaises, qui garde en Europe son irréductible spécificité de système macro-céphale. Faut-il le regretter? Faut-il tenter de faire évoluer ce système vers un modèle de type rhénan? Quels sont les comportements des acteurs qu'il conviendrait de modifier pour aller dans ce sens? Les processus à mettre en œuvre sont-ils compatibles avec les formes actuelles de la mise en réseau des villes à l'échelle européenne? Sans mesure, sans outil de modélisation, il est bien évident que personne ne peut répondre de l'effet d'une intervention dans un système aussi complexe. Les connaissances relatives aux systèmes de villes ne sont pas assez assurées pour que les acteurs sachent non seulement s'y adapter, mais encore les modifier, et les modèles disponibles, avec ou sans acteurs, sont encore bien trop incertains et fragmentaires pour guider l'action.

\section{CONCLUSION}

Ce sont ainsi deux types de préoccupations bien différentes qui inciteraient à observer de plus près quels sont les comportements individuels, dont l'agrégation produit autant de régularités à l'échelle des système de villes. D'une part, s'ils veulent que leur contribution puisse s'articuler à celles d'autres sciences sociales, les géographes ont intérêt à compléter leur démarche habituelle, inspirée par un certain «holisme méthodologique» (Valade, 2001), en traduisant les régularités qu'ils observent en termes de comportements d'acteurs - ce qui n'implique pas nécessairement de comprendre les intentions de responsables en interaction, tâche qui peut être laissée aux spécialistes de sciences politiques, mais ce qui suppose au moins de se livrer à des analyses «multi-niveaux » plus systématiques. D’autre part, leur rôle actif de conseillers dans l'aménagement du territoire ne peut demeurer crédible que s'il s'appuie sur des interprétations reliant de manière explicite des évolutions macrogéographiques, non plus aux seules interactions entre des entités mésogéographiques, mais aussi à des comportements individuels.

Université Paris I

pumain@parisgeo.cnrs.fr 


\section{RÉFÉRENCES}

Archaeomedes (ed) 1998, Des oppida aux métropoles. Paris, Anthropos, coll. Villes, 290 p.

Berry B.J.L., 1964, Cities as systems within systems of cities, Papers of the Regional Science Association, 13, 147-163.

Bretagnolle A. 1999, Espace et temps dans les systèmes de villes. Université Paris I, thèse de doctorat.

Bura S. Guérin-Pace F. Mathian H. Pumain D. Sanders L. 1996, Multi-agent systems and the dynamics of a settlement system. Geographical Analysis, 2, 161-178.

Ferber J. 1995, Les systèmes muti-agents : vers une intelligence collective. Paris, Interédition.

Gibrat R. 1931, Les inégalités économiques. Paris, Sirey.

Guérin-Pace F., Mathian H. Pumain D. Sanders L. Bura S. 1996, Les systèmes multi-agents pour modéliser l'émergence des réseaux urbains, in Bocquet-Appel J.P., Courgeau D. et Pumain D. Analyse spatiale de données biodémographiques, John Libbey-INED, 281-309.

Mackinder H.J. 1902, Britain and the British Isles. London, W. Heinemann.

Pred A. 1977, City systems in advanced societies. London, Hutchison.

Pumain D. 1982, La dynamique des villes. Paris, Economica.

Pumain D. 1997, Vers une théorie évolutive des villes. L’Espace Géographique, 2, 119-134.

Pumain D. Robic M.-C. 1996, Théoriser la ville, in Derycke P. Huriot J.M. Pumain D., Penser la ville, Paris, Anthropos, chap. 4, 107-161.

Reclus E. 1977, La France (Nouvelle Géographie Universelle, la Terre et les Hommes, t. II). Paris, Hachette.

Robic M.C. 2001, Walter Christaller et la théorie des «lieux centraux», in Lepetit B. Topalov C. (dir.) $L a$ ville des sciences sociales, Paris, Belin, 151-189.

Valade B. 2001, De l'explication dans les sciences sociales: holisme et individualisme, in Berthelot J.-M. (ed) Epistémologie des sciences sociales. Paris, PUF, 357-405. 